\title{
Effect of Aqueous Extract of Dendropanax morbifera Leaf on Sexual Behavior in Male Rats
}

\author{
Myung-A Jung ${ }^{1,2}$, Kyo-nyeo Oh ${ }^{1}$, Eun Jin Choi ${ }^{1}$, Yu jin Kim ${ }^{1}$, Donghyuck Bae ${ }^{1}$, Dool-Ri Oh${ }^{1}$, Ki man Kim ${ }^{3}$, \\ Dong-Wook Kim², Chul-yung Choi ${ }^{1, *}$ \\ ${ }^{1}$ Jeonnam Bioindustry Foundation, Jeonnam Insittute on Natural Resources Research, Jeollanamdo, South Korea \\ ${ }^{2}$ Department of Oriental Medicine Resources, College of Natural Science, Mokpo National University, Jeollanamdo, South Korea \\ ${ }^{3}$ Serom Co., Ltd., Jeollanamdo, South Korea \\ *Corresponding author: blockstar@hanmail.net
}

\begin{abstract}
Sexual function in an important of the quality of life and subjective well-being in humans. Successful treatment of sexual dysfunction may improve not only sexual relationships, but also overall quality of life. The objective of this study was to determine the effects of Dendropanax morbifera (DM) Lev. leaf extracts on sexual behavior and testosterone levels in male rats. Three different doses of DM $(50,100$, and $200 \mathrm{mg} / \mathrm{kg} / \mathrm{day})$ were administered orally for 28 days. On day 28 (final day of the sample treatment period) and day 42 (after 2 weeks of sample withdrawal period following 28 days of sample treatment), serum testosterone levels and sexual behavior of rats were determined, including mount frequency (MF), intromission frequency, mount latency (ML, the time from introduction of the female to the male to the first mount), intromission latency (IL), and post-ejaculatory interval (PEI). ML significantly reduced, whereas MF increased in the DM group. The DM group also had a reduced PEI. The oral administration of DM significantly increased serum testosterone levels compared with those in the control group. The results of this study demonstrate that an aqueous extract of DM represents a useful natural resource for the management of sexual function.
\end{abstract}

Keywords: sexual behavior, testosterone, erectile dysfunction, Dendropanax morbifera

Cite This Article: Myung-A Jung, Kyo-nyeo Oh, Eun Jin Choi, Yu jin Kim, Donghyuck Bae, Dool-Ri Oh, Ki man Kim, Dong-Wook Kim, and Chul-yung Choi, "Effect of Aqueous Extract of Dendropanax morbifera Leaf on Sexual Behavior in Male Rats." Journal of Food and Nutrition Research, vol. 5, no. 7 (2017): 518-521. doi: $10.12691 /$ jnr-5-7-10.

\section{Introduction}

Sexual function is an important component of quality of life and subjective well-being in humans. Erectile dysfunction (ED) is one of the most common sexual dysfunctions in men [1]. The association between androgens and erectile function at the peripheral level has been established [2,3], with androgens acting on the central nervous system to serve as important modulators of male sexual behavior, including libido. The frequency of ejaculation and sexual intercourse correlate with serum testosterone levels in the normal to subnormal range [4]. Sexual problems are widespread, and can adversely affect mood and interpersonal functioning [5]. Male sexual dysfunction (MSD) has various etiologies, including personal lifestyle, androgen deficiency, aging, psychological disorders; as a side effect of antihypertensive, psychiatric, or antiulcer medications, antidepressants, or antiandrogens; as well as chronic medical conditions such as diabetes, hypertension, and pulmonary cancer [6]. In general, the mechanisms leading to normal erectile function imply inter-connections among neurons, striated perineal muscles, and androgens, which are responsible for maintaining sexual behavior in adults. or several hundred years, men have used locally grown plants as supplements to energize, vitalize, and ultimately improve sexual function.

Dendropanax morbifera is a subtropical, broad-leaved evergreen tree belonging to the family Araliaceae. Lacquer from DM has a golden color and is used as a varnish in woodworking and metalworking [7]. DM is an endemic species in South Korea, found naturally only in southern regions [8]. It has also been cultivated on Jindo Island, Suncheon City, and Wando Island in South Korea. Several parts of DM are used in folk medicine for the treatment of migraines and dysmenorrhea [9]. DM Leveille extracts have been reported to exert various effects, including immune activation against cancer cells, antioxidant effects [10,11] anti-diabetic [12] and insecticidal effects [13]. In addition, its anti-complement [14] and antibiotic effects on liver diseases have been studied. This study therefore evaluated the effect of DM on sexual behavior and serum testosterone levels in male rats.

\section{Materials and Methods}

\subsection{Preparation of Extracts from DM}

DM was obtained from Jindo, Jeollanamdo, South Korea, and was washed with water three times and dried 
at $45^{\circ} \mathrm{C}$ in a drying oven for $48 \mathrm{~h}$ before being extracted with 20 volumes of distilled water at $100^{\circ} \mathrm{C}$ for $3 \mathrm{~h}$. The residue was removed by filtration using a $150-\mathrm{mm}$ mesh, and the filtrate was evaporated at 16 Brix and freeze-dried to obtain a powder of DM. The dried extract was stored at $4^{\circ} \mathrm{C}$ to avoid degradation.

\subsection{Experimental Design}

Male and female 5-week-old Wistar rats were purchased from Samtako (Osan, South Korea). The rats were kept in plastic cages under controlled temperature $\left(23 \pm 2^{\circ} \mathrm{C}\right)$ and humidity $\left(50 \pm 5^{\circ} \mathrm{C}\right)$, with $100 \%$ fresh HEPA-filtered air, and a 12-h light-dark cycle (lights on from 08:00 to 20:00). All experimental procedures were conducted in accordance with guidelines for the care of experimental animals as approved by the Institute of Animal Care and Use Committee of Jeollanamdo Institute for Natural Resources Research (approval number JINR-1415). The rats were provided ad libitum access to basal diet and water during experiments. The rats were divided into four groups of five each as follows: no administration of DM (control); $50 \mathrm{mg} / \mathrm{kg}$ of DM administered group (DM 50); $100 \mathrm{mg} / \mathrm{kg}$ of DM administered group (DM 100); and $200 \mathrm{mg} / \mathrm{kg}$ of DM administered group (DM 200). The extracts were dissolved in distilled water and orally administered daily for 4 weeks. The rats were provided ad libitum access to basal diet and water during the experiment.

\subsection{Sexual Behavior Test}

Female rats were injected subcutaneously with $10 \mu \mathrm{g} / 100 \mathrm{~g}$ body weight estradiol benzoate and $0.5 \mathrm{mg} / 100 \mathrm{~g}$ body weight progesterone 48 and $4 \mathrm{~h}$, respectively, before the mating day ( $28^{\text {th }}$ day of extract treatment). Sexual behavior was monitored in a separate room for $30 \mathrm{~min}$. Ten minutes was allocated for adaptation, after which a primed female was placed in a cage with a male. On day 28 (final day of sample treatment period) and day 42 (after 2 weeks of sample withdrawal period following 28 days of sample treatment), the sexual behavior of the rats was observed. The following parameters of sexual behavior were measured as previously described [15]: (1) Mount latency (ML): time from the introduction of the female until the first mount. (2) Mount frequency (MF): number of mounts preceding ejaculation. (3) Intromission latency (IL): time from introduction of the female until the first intromission (vaginal penetration). (4) Intromission frequency (IF): number of intromission preceding ejaculation. (5) Postejaculatory interval (PEI): time from ejaculation until the next intromission.

\subsection{Serum Testosterone Levels}

Blood samples were collected on day 28 and day 42 and serum testosterone levels were measured using a testosterone EIA kit (ENZO life science, Farmingdale, USA) according to the manufacturer's instruction.

\subsection{Statistical Analysis}

The results are expressed as the mean \pm standard error. The data were statistically evaluated using a one-way analysis of variance (ANOVA) followed by Duncan's multiple comparison test using SPSS software (Windows version 17, IBM Corporation, New York, USA). The statistical significance was defined at $\mathrm{P}<0.05$.

\section{Results}

\subsection{Effect of DM on Sexual Behavior in Male Rats}

The enhancement of libido may be due to an increase in the anterior pituitary hormone and serum testosterone levels, which in turn stimulates sexual behavior. To investigate sustaining power, which is the ability for continuation of improved sexual function, we evaluated sexual behavior on day 14 after the withdrawal of DM treatment according to specific parameters of sexual behavior. ML evaluated on day 28 (final day of the sample treatment period) and day 42 (after 2 weeks of sample withdrawal period following 28 days of sample treatment) of treatment is shown Figure 1. ML significantly decreased in the DM 200 group $(14.9 \pm 1.88 \mathrm{~s})$ compared with that in the control group. MF evaluated on days 28 and 42 of treatment is shown in Figure 2, and was found to be significantly increased in the DM 200 group $(67.9 \pm 1.17)$ on day 28 , although no significant change was observed on day 42. IL decreased in the DM 200 group $(40.5 \pm 6.85 \mathrm{~s})$ on day 28 compared with that in the control group. In the DM 200 group, IL decreased significantly on day 42 of DM treatment (Figure 3). IF increased in the DM 100 and DM 200 groups when compared with that in the control group. At all three doses, DM showed no significant effect on IF on days 28 and 42 of treatment (Figure 4). PEI decreased in the DM 200 group compared with that in the control group (Figure 5). However, at all three doses, DM showed no significant effect on PEI on days 28 and 42 of treatment. These results suggest that DM treatment may be responsible for the prolongation of improved sexual function after its withdrawal.

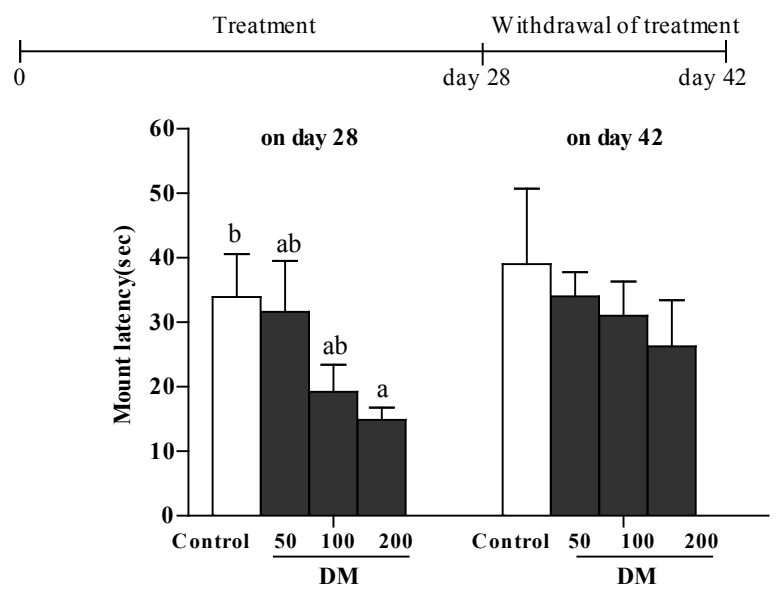

Figure 1. Effect of DM on mount latency in male rats. Control, no administration of DM; DM 50, $50 \mathrm{mg} / \mathrm{kg}$ DM administered group; DM $100,100 \mathrm{mg} / \mathrm{kg}$ DM administered group; DM 200, $200 \mathrm{mg} / \mathrm{kg} \mathrm{DM}$ administered group. Values are expressed as the mean \pm SE. Different letters above the bars indicate significant differences by Duncan's multiple comparison tests $(\mathrm{P}<0.05)$ 

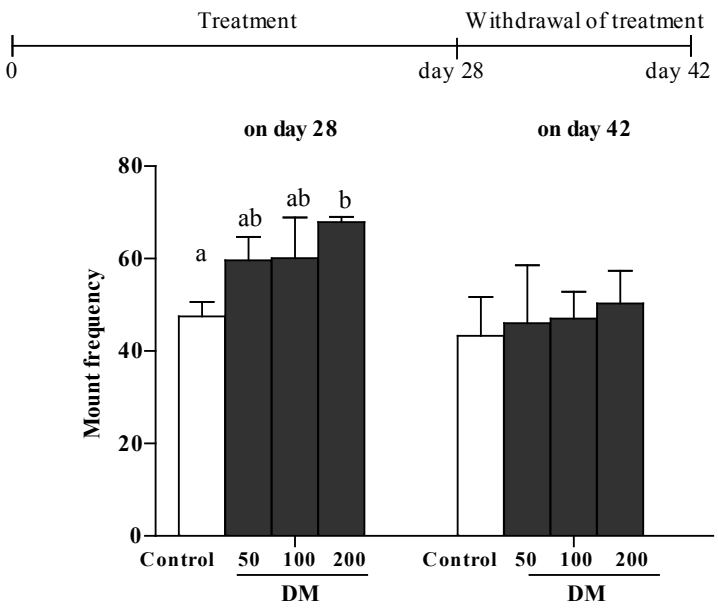

Figure 2. Effect of DM on mount frequency in male rats. Control, no administration of DM; DM $50,50 \mathrm{mg} / \mathrm{kg}$ DM administered group; DM $100,100 \mathrm{mg} / \mathrm{kg}$ DM administered group; DM 200, $200 \mathrm{mg} / \mathrm{kg}$ DM administered group. Values are expressed as the mean \pm SE. Different letters above the bars indicate significant differences by Duncan's multiple comparison tests $(\mathrm{P}<0.05)$

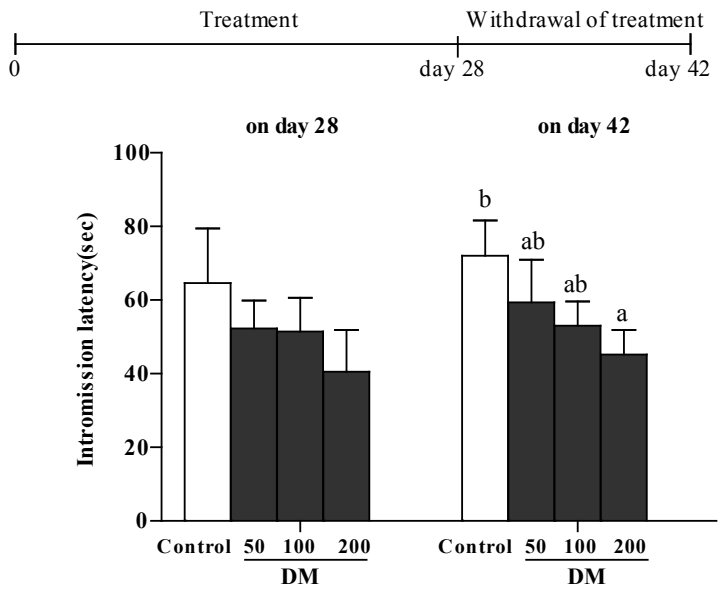

Figure 3. Effect of DM on intromission latency in male rats. Control, no administration of DM; DM $50,50 \mathrm{mg} / \mathrm{kg}$ DM administered group; DM $100,100 \mathrm{mg} / \mathrm{kg}$ DM administered group; DM 200, $200 \mathrm{mg} / \mathrm{kg}$ DM administered group. Values are expressed as the mean \pm SE. Different letters above the bars indicate significant differences by Duncan's multiple comparison tests $(\mathrm{P}<0.05)$
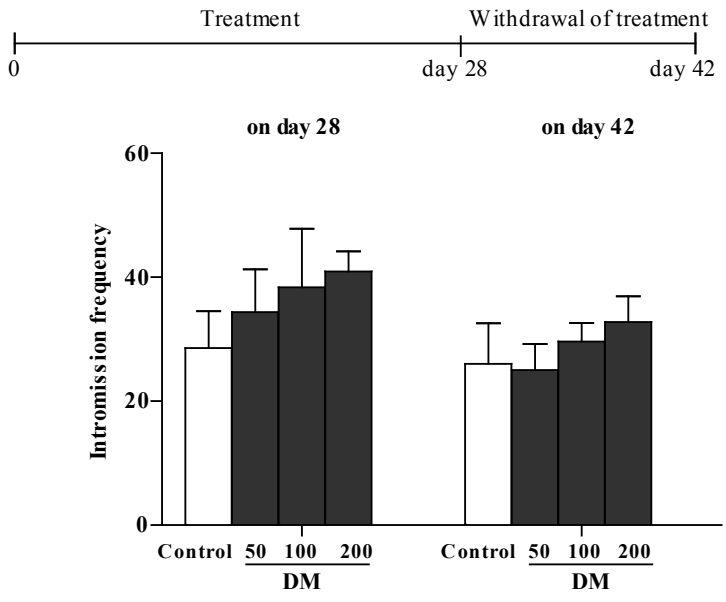

Figure 4. Effect of DM on intromission frequency in male rats. Control, no administration of DM; DM $50,50 \mathrm{mg} / \mathrm{kg}$ DM administered group; DM 100, $100 \mathrm{mg} / \mathrm{kg}$ DM administered group; DM 200, $200 \mathrm{mg} / \mathrm{kg}$ DM administered group. Values are expressed as the mean $\pm \mathrm{SE}$
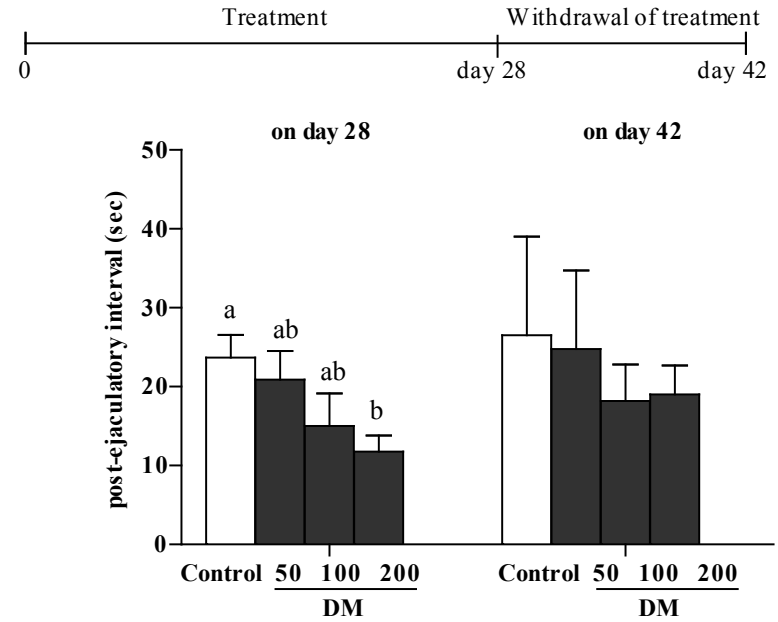

Figure 5. Effect of DM on postejaculatory interval in male rats. Control, no administration of DM; DM 50, $50 \mathrm{mg} / \mathrm{kg}$ DM administered group; DM 100, $100 \mathrm{mg} / \mathrm{kg}$ DM administered group; DM 200, $200 \mathrm{mg} / \mathrm{kg}$ DM administered group. Values are expressed as the mean \pm SE. Different letters above the bars indicate significant differences by Duncan's multiple comparison tests $(\mathrm{P}<0.05)$

\subsection{Serum Testosterone Levels}

The testosterone levels evaluated on days 28 and 42 of DM treatment are shown in Figure 6. Testosterone significantly increased in the DM 200 group $(2.0 \pm$ $0.04 \mathrm{ng} / \mathrm{mL}$ ), whereas no significant change in testosterone level was observed on day 42 of treatment.
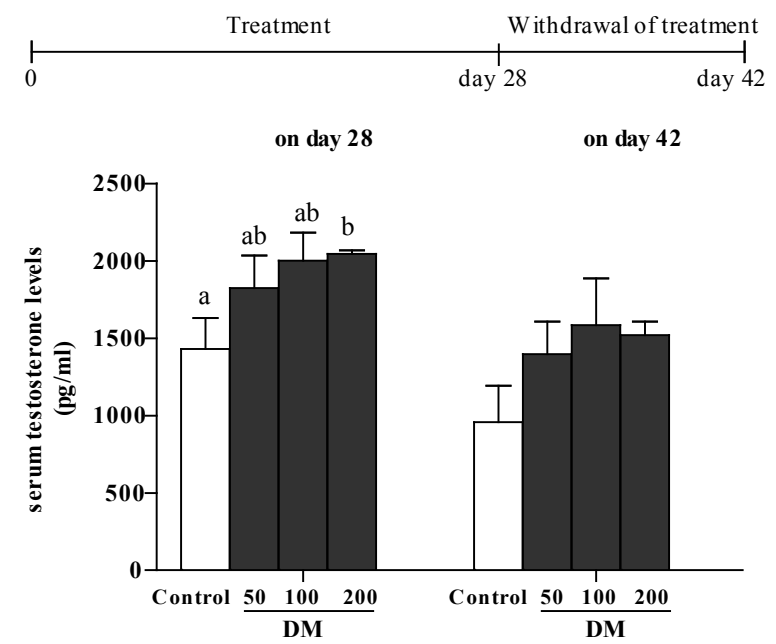

Figure 6. Effect of DM on testosterone levels in male rats. Control, no administration of DM; DM $50,50 \mathrm{mg} / \mathrm{kg}$ DM administered group; DM $100,100 \mathrm{mg} / \mathrm{kg}$ DM administered group; DM 200, $200 \mathrm{mg} / \mathrm{kg}$ DM administered group. Values are expressed as the mean \pm SE. Different letters above the bars indicate significant differences by Duncan's multiple comparison tests $(\mathrm{P}<0.05)$

\section{Discussion}

Androgen acts on the central nervous system to serve as an important modulator of male sexual behavior including libido. Sexual behavior is dependent on the normal functioning of the hypothalamic-pituitary-gonadal axis [16]. DM treatment at $200 \mathrm{mg} / \mathrm{kg}$ body weight enhanced the sexual behavior of male rats, producing better results than at 50 and $100 \mathrm{mg} / \mathrm{kg}$ body weight. MF and IF are 
useful indices of vigor, libido, and potency. While MF reflects sexual motivation, increase in IF indicates the efficiency of erection, penile orientation, and the ease with which ejaculatory reflexes are activated [15]. In this study, the increase in IF due to DM treatment suggests that the mechanism of penile erection was activated. ML and IL are indicators of sexual motivation. They are inversely proportional to sexual motivation. Therefore, a decrease in ML observed at $200 \mathrm{mg} / \mathrm{kg}$ body weight of the extract on day 28 may imply the stimulation of sexual motivation and arousal. It may also indicate enhanced sexual appetitive behavior in the male rats. All these findings further support the potential of the extract in improving sexual function at these doses. PEI is considered an index of potency, libido, and the rate of recovery from exhaustion after the first series of mating [17]. A PEI more than $5400 \mathrm{~s}$ indicates that the male is sexually exhausted and the intensity of sexual behavior will be reduced in subsequent mating [15]. Therefore, the decreased PEI at $200 \mathrm{mg} / \mathrm{kg}$ DM treatment on day 28 can be attributed to enhanced potency and libido or less exhaustion in the first series of mating or both. Testosterone and its metabolites play a key role in sexual function and are closely associated with ED as well as sexual desire and activity. This demonstrates the potential of testosterone therapy in the treatment of ED. The increase in serum testosterone levels on day 28 induced by the administration of $200 \mathrm{mg} / \mathrm{kg} \mathrm{DM}$ may be responsible for the enhanced sexual behavior in the present study. The sexual behavior and serum testosterone levels showed improvement on day 42 of the treatment. The outcome of this study indicates that the effects of DM in improving sexual behavior are sustained even after withdrawal of the treatment. The enhancement of libido may be due to an increase in the anterior pituitary hormone and serum testosterone levels, which in turn stimulates sexual behavior $[18,19]$. Further studies will be required to elucidate the mechanism underlying the association between sexual behavior and androgens.

\section{Conclusion}

The present study demonstrated that DM could enhance sexual behavior in male rats. The results of the present study suggest that DM enhances various indicators associated with sexual function and has the potential to be used as a natural substance in the treatment of ED.

\section{Acknowledgements}

The research was financially supported by the Korean Institute of Planning \& Evaluation for Technology in Food, Agriculture, Forestry \& Fisheries (IPET) through the High Value-Added Food Technology Development Program, funded by Ministry of Agriculture, Food and Rural Affairs (MAFRA) (114024-03-3-HD020).

\section{References}

[1] Wagner, G. and Saenz de, T.I. Update on male erectile dysfunction, BMJ 316 (1998) 678-682

[2] Morelli, A., Filippi, S., Vignozzi, L., Mancina, R. and Maggim, M. Physiology of erectile function: an update on intracellular molecular processes, EAU-EBU Update Series, 2006, (4) 96-108.

[3] Wallen, K., and Hassett, J.M. Sexual differentiation of behavior in monkeys: role of prenatal hormones, J. Neuroendocrinol, 2009. 151, 595-605

[4] Nieschlag, E. and Behre, H.M. Testosterone: action, deficiency, substitution. fourth ed., Springer Verlag, Berlin

[5] Shin, B.C., Lee, M.S., Yang, E.J., Lim, H.S. and Ernst, E. Maca (L. meyenii) for improving sexual function: a systematic review. BMC Complement. Altern. Med. 2010. 10 (44), 1-6.

[6] Yakubu, M.T., Akanji, M.A. and Oladiji, A.T. Male sexual dysfunction and methods used in assessing medicinal plants with aphrodisiac potentials, Pharmacognosy Review. 2007. 1, 49-56.

[7] Kim, S.H., Jang, Y.S., Han, J.G., Chung, H.G., Lee, S.W. and Cho, K.J. Genetic variation and population structure of Dendropanax morbifera Lev. (Araliaceae) in Korea. Silvae Genet. 2006. 55, 7-13.

[8] Han, S.H., Jung, T.H., Oh, M.H., Ko, M.H., Oh, Y.S., Koh, S.C., Kim, M.H. and Oh, M.Y. Phytogenetic relationships of the Dendropanax morbifera and D. Trifidus based on PCR-RAPD. Kor. J Genetics. 1998. 20, 173-181.

[9] Bae, K.H. The medical plants of Korea, Kyo-Hak publishing Seoul. 2000.

[10] Lee, B.H., Choi, J.H. and Kim, H.J. Curing and thermal behaviors of Korean Dendropanax Lacquer made by acetone and wine spirit extraction methods, Prog. Org. Coatings. 2002. 52, 241-245.

[11] Hyun, T.K., Kim, M.O., Lee, H.K., Kim, Y.J., Kim, E.K. and Kim, J.S. Evaluation of anti-oxidant and anti-cancer properties of Dendropanax morbifera Leveille, Food Chem. 2013. 141, 1947-1955.

[12] Moon, H.I. Antidiabetic effects of dendropanoxide from leaves of Dendropanax morbifera Leveille in normal and streptozotocininduced diabetic rats, Hum. Exp. Toxicol. 2011. 30, 870-875.

[13] Chung, I.M., Kim, M.V., Park., W.H. and Moon, H.I. Antiaterogenic activity of Dendropnax morbifera essential oil in rats, Pharmazie 2009. 64, 547-549.

[14] Park, B.Y., Min, B.S., Oh, S.R., Kim, J.H., Kim, T.J., Kim, D.H., Bae, K.H. and Lee, H.K. Isolation and anticomplement activity of compounds from Dendropanax morbifera, J. Ethnopharmacol. 2004. 90, 2004, 403-408.

[15] Agmo, A. Male rat sexual behavior, Brain Res. Protoc. 1997. 1, 203-209.

[16] Andersson, K.E. Pharmacology of penile erection, Pharmacol. Rev. 2001. 53, 417-450.

[17] Tajuddin, A., Ahmad, S., Latif, A. and Qasmi, I.A. Effect of 50\% ethanolic extract of Syzygium aromaticum (L.) Merr. \& Perry. (clove) on sexual behaviour of normal male rats, BMC Complement. Altern. Med. 2004. 4, 17-24.

[18] Taha, S.A., Islam, M.W. and Ageel, A.M. Effect of ambrein, a major constituent of ambergris, on masculine sexual behavior in rats, Arch. Int. Pharmacodyn. Ther. 1995. 329, 283-294.

[19] Subramoniam, A., Madhavachandran, V., Rajasekharan, S. and Pushpangadan, P. Aphrodisiac property of Trichopus zeylanicus extract in male mice, J. Ethnopharmacol. 1997. 57, 21-27. 